\title{
The study of pulsating stars from the COROT exoplanet field data
}

\author{
C. Aerts \\ Instituut voor Sterrenkunde, Catholic University of Leuven, Belgium
}

\begin{abstract}
In this paper we present our proposal to search for periodically variable stars in the exoplanet field data. In order to do so one first has to perform a quasiautomated frequency search in the data. Once the periodically variable stars are found, they have to be classified, e.g. by means of a statistical scheme analoguous to the one we developed for the classification of new B-type variables discovered from the HIPPARCOS data.

After the classification of the variable stars, we plan to study the $\beta$ Cep stars and the slowly pulsating B stars (SPBs) with the goal to derive constraints on their internal structure.
\end{abstract}

\section{Goal of the project}

In this project we propose to make full use of the COROT exoplanet field data for asteroseismology. The observation strategy adopted by the planet hunters is ideally suited for asteroseismology of some classes of pulsators, i.e. this project is a free by-product of the search for planets within the COROT framework. The individual integration times of 32 seconds for the CCD frames of the exoplanet field will in any case result in a time sampling of the order of minutes during 5 consecutive months. This is largely sufficient to derive very accurately the pulsation frequencies for several types of $\kappa$ driven modes in main-sequence stars, such as the $\beta$ Cep stars, the slowly pulsating B stars (hereafter termed SPBs), the $\gamma$ Doradus stars and most $\delta$ Scuti stars. The expected photometric accuracy will be at least an order of magnitude higher than the one of current groundbased datasets of such types of stars.

COROT is by far better suited to perform our project than MOST and MONS. MOST will observe only a few very bright stars, while the MONS 
Field Monitor will gather data for a few thousand stars, which will be observed during one month. COROT is therefore the only mission resulting in data with a total time base significantly longer than a month. Particularly, main-sequence gravity-mode pulsators, i.e. $\gamma$ Doradus stars and SPBs, cannot be studies by MOST and MONS, as they have beat periods of several months or even years.

The concrete goal of our project is twofold: 1) the search for, and classification of, new variable stars; 2) the derivation of detailed frequency spectra of the new variables and their physical interpretation.

\section{Search for new variables}

The discovery of new variables in the COROT exoplanet fields will be quite straightforward in view of the excellent time sampling. Such high temporal resolution is beyond comparison with e.g. the one of the HIPPARCOS mission. The long time base of the data of the latter mission already allowed the discovery of thousands of new periodically variable stars, despite the relatively poor sampling (Eyer, 1998). This led to the publication of the HIPPARCOS catalogue of periodic variable stars (van Leeuwen et al. 1997, Grenon et al. 1997).

A considerable amount of new variables will be found among the 30000 stars which will all be monitored during 5 months by COROT. The application of classical period search algorithms will result in the detection of many new periodically variable stars of different kinds. In view of the high-temporal resolution and the high duty cycle, Fourier analysis will suffice to perform the frequency search.

\section{Classification of the new variables: discriminant analysis}

The newly discovered periodic variables need to be classified, i.e. the origin of their variability has to be decided upon. Waelkens et al. (1998) have developed a classification scheme for the new HIPPARCOS B-type variables. Their method is based upon multivariate discriminant analysis. With this method, one makes use of different known parameters of the stars. One of these parameters is of course the frequency. Besides this quantity, Waelkens et al. (1998) used the Geneva parameters X, Y, Z. These parameters are reddening-free quantities derived from the Geneva colours. Since the COROT exoplanet data will contain three colours, the method developed by Waelkens et al. is also applicable to the COROT data after minor modification. It was also already successfully applied by Aerts et al. (1998) in their search for new $\gamma$ Doradus candidates from the HIPPARCOS data. 
The essential ingredients of discriminant analysis are the following: In general, multivariate classification schemes use the knowlegde of stellar parameters in an $n$-dimensional space. The classification of new variables is best done in two steps: the assignment of a new variable to a known class according to a distance measure and subsequently a check through bivariate plots. Discriminant analysis is only possible when prototypes of classes are available, such that a "definition" of a known class can be formulated. The unknown objects will then be assigned to one of these so-called calibration classes. This assignment is obtained by the determination of discriminating axes in the multidimensional space, in such a way that optimal separation of the predefined classes is attained. For each star the Mahalanobis or generalised distance to the discriminating axes is used for assignment to one of the definition classes. However, stars with a relatively large Mahalanobis distance still might have a different origin of variability than those of the class to which they were assigned. A definite classification is then made by means of bivariate plots within the $n$-dimensional parameter space. The stars with a large Mahalanobis distance having also another position in bivariate plots compared to the calibration stars are probably of a different nature and cannot be assigned to one of the calibration classes. A typical example could be a Be star, a CP star or a supergiant. For these stars it is often not possible to provide a clear definition of the class as there are e.g. very different timescales in the variability of Be stars, there are supergiants with and without microvariations, etc. It is better not to use them as calibration classes. All new variables of such kind will then at first be assigned to a wrong class but their different nature will be derived from the large Mahalanobis distance and/or a deviating position in the bivariate plots.

Before applying the discriminant analysis one needs to choose the parameters to be used for the definition of the classes. In the framework of our proposal these are the frequency and the three COROT colours. This implies that we have to determine the COROT colours for the known variables of the different types of pulsators, in order to find the definition of each class.

\section{Interpretation of the variability}

A next step in our project consists of the detailed interpretation of the frequencies in terms of asteroseismology. In order for this to be possible, we are obliged to limit ourselves to the multiperiodic variables with correct mode identification. The latter remains a serious problem for $\kappa$ driven modes outside the asymptotic regime. As we will be dealing with faint stars having long beat periods, spectroscopic mode identification methods will not be of any help. The best way to identify modes from frequency spectra is in that circumstance the detection of rotationally- or magnetically-induced frequency multiplets. 


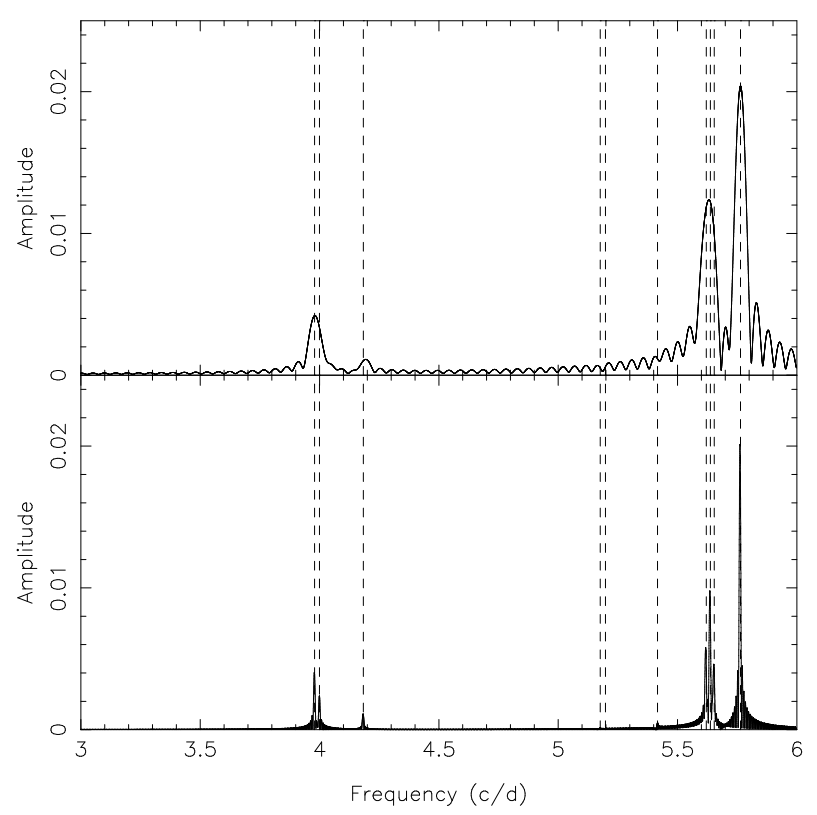

Figure 1: Frequency analysis of the simulated signal for the typical $\beta$ Cep star with a time span of 20 days (top) and of 5 months (bottom). The dashed lines indicate the input frequencies.

We have performed some preliminary simulations in order to derive the capabilities of COROT with respect to the detection of multiple periods. In view of our expertise we have limited the simulation study to pulsating B stars, i.e. $\beta$ Cep stars and SPBs. We have simulated a periodic signal typical for a $\beta$ Cep star and an SPB respectively. For none of these stars the mode selection mechanism is understood and we have no idea how many modes are excited. The WIRE data of $\beta$ Crucis have clearly indicated that more modes are excited than thought up to now from ground-based photometry in this $\beta$ Cep star (Cuypers et al. 2002). This is also the case for the $\delta$ Scuti star $\theta^{2}$ Tauri (Poretti et al. 2002). Taking these recent results into account we have selected 10 frequencies out of the pool of observed frequencies for the two classes of stars for our simulation. For the prototypical $\beta$ Cep star it concerns frequencies between 3 and 6 $\mathrm{cd}^{-1}$ and for the SPB between 0.3 and $0.8 \mathrm{~cd}^{-1}$. We have included observed doublet and triplet frequencies. The amplitudes were taken between $50 \mu \mathrm{mag}$ and $20 \mathrm{mmag}$, which is the maximum amplitude observed for such stars. No noise was added at this stage. 


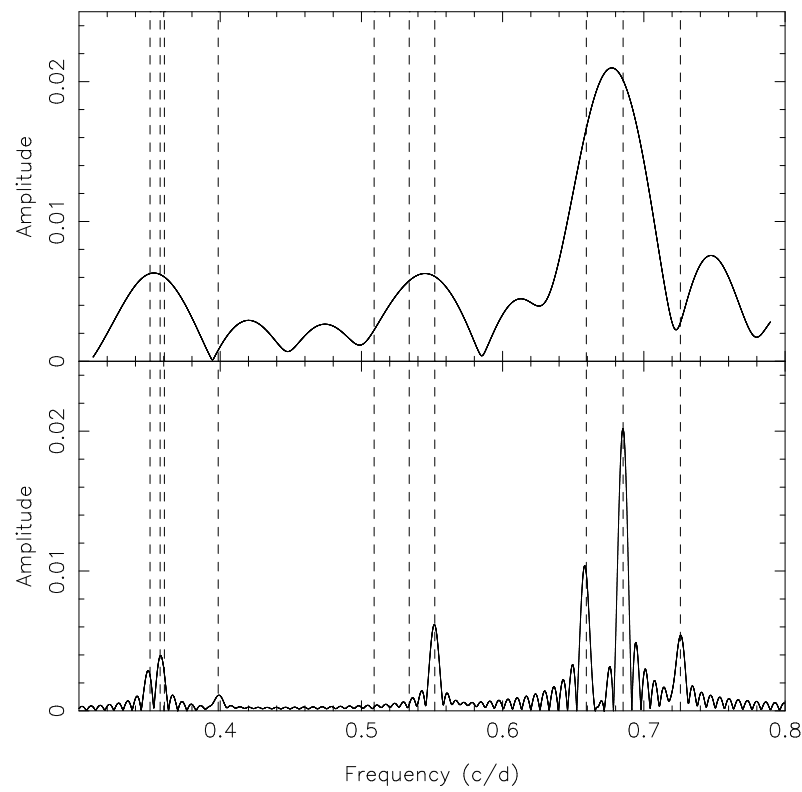

Figure 2: Frequency analysis of the simulated signal for the typical SPB with a time span of 20 days (top) and of 5 months (bottom). The dashed lines indicate the input frequencies.

We generated light curves with the following two different time samplings:

1. every 3 minutes during 20 days,

2. every 20 minutes during 5 months.

Such sampling is quite conservative as COROT should do better.

The Fourier spectra for the simulated signals of the $\beta$ Cep star, respectively SPB, are shown in Figs. 1 and 2. We are particularly interested to see whether COROT would be able to result in the detection of the multiplets. This will not be the case for the data obtained during the exploratory phases, as can be derived from the upper panels of Figs. 1 and 2. The runs of 5 months do result easily in the derivation of the multiplet frequencies of the $\beta$ Cep star, which is very important. This will imply that the COROT data will in principle allow us to derive the internal rotation law in stars that will end their lives as type II supernovae. Most of the close frequencies of the SPB are also recovered, albeit not all of them. This corresponds to the well-known fact that the beat periods of such stars can be of the order of a year. At least 7 of the 10 frequencies are easily recovered, though, in our exercise. 
Of course, our very basic simulations have to be redone, by convolving the generated signal with a realistic noise-budget valid for COROT. This will be done in a forthcoming study. However, the simple exercise allows us to derive the following conclusions :

- the time span of the COROT exploratory programme is too short to disentangle the frequencies of pulsating $B$ stars;

- rotational splittings will easily be found for $\beta$ Cep stars in the SISMO core programme, as the sampling is much better than the one we have taken in this study; the core programme will also allow the detection of a very large fraction of the excited gravity modes of SPBs;

- the exoplanet data are ideally suited for detecting the larger-amplitude modes of $\beta$ Cep stars and of SPBs.

All the conclusions for the SPB-type pulsations are also valid for the gravity modes in $\gamma$ Doradus stars. We did not include such stars in the simulation as the observational inventory available for them does not reach the same level of completeness as the ones of the $\beta$ Cep stars and SPBs.

\section{Future work}

We have already stressed the potential of the COROT exoplanet data for asteroseismology of massive stars at previous COROT meetings. With our basic study provided here, we have shown that our goal is indeed feasible. In the near future we will perform a more extensive simulation study with a realistic noise budget. We will particularly concentrate on the derivation of the detection limit of modes for the exoplanet data. One of the other main goals of this future simulation study is to derive the optimal rebinning for the analysis of gravity modes in SPBs and $\gamma$ Doradus stars.

\section{References}

Aerts, C., Eyer, L., Kestens, E. 1998, A\&A 337, 790

Cuypers, J., Aerts., C., Buzasi, D., Catanzarite, J., Conrow, T., Laher, R. 2002, in

ASP Conf. Ser., Vol. 259, Radial and Nonradial Pulsations as Probes of Stellar

Physics, eds. C. Aerts, T.R. Bedding and J. Christensen-Dalsgaard,

(San Francisco ASP), in press

Eyer, L. 1998, PhD Thesis, Geneva Observatory

Grenon, M. et al. 1997, ESA SP-1200, Volume 12

Poretti, E., Buzasi, D., Laher, R., Catanzarite, J., Conrow, T. 2002, A\&A, in press (astro-ph/0111361)

van Leeuwen, F. et al. 1997, ESA SP-1200, Volume 11

Waelkens, C., Aerts, C., Kestens, E., Grenon, M., Eyer, L. 1998, A\&A 330, 215 